
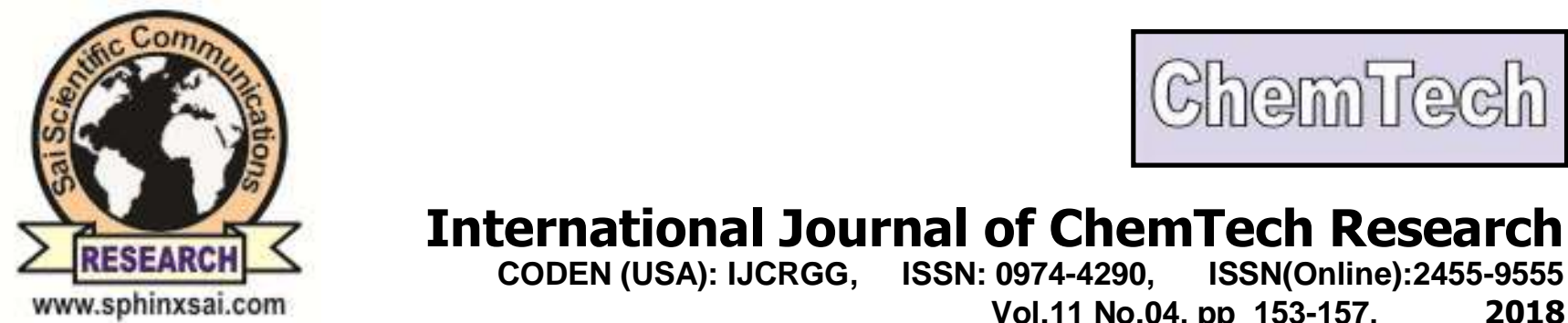

International Journal of ChemTech Research CODEN (USA): IJCRGG, ISSN: 0974-4290, ISSN(Online):2455-9555

Vol.11 No.04, pp 153-157,

2018

\title{
Steady State Optimal Power Flow
}

\author{
${ }^{\star 1}$ Ravi.C.N., ${ }^{2}$ Lakshmi.D., ${ }^{3}$ Benisha.S, ${ }^{4}$ Zahira.R. \\ 'Dept of EEE, Vidya Jyothi Institute of Technology, Hyderabad -500075, India \\ ${ }^{2,3}$ Dept of EEE, Sree Sastha Institute of Engineering \& Technology, \\ Chennai-600123, India. \\ ${ }^{4}$ Dept of EEE, Tagore Engineering College, Chennai, India
}

\begin{abstract}
An optimal load-flow solution gives the optimal active and reactive power dispatch for a static power system loading condition. Computationally, it is a very demanding nonlinear programming problem, due to the large number of variables and in particular to the much larger number of variables and in particular to the much larger number and types of limit constraints which define the boundaries of technical feasibility This paper solves the standard 26 bus power system network.
\end{abstract}

Keywords : optimal power flow; OPF; Lamda iteration

\section{Introduction}

One of most important and frequently used analysis and computational procedures for the planning, design, and operation of an electric power system is the ac power-flow program. This program constitutes a simulation of the steady-state ac power flows and voltages in the network under study. It is used to simulate the flows and voltages corresponding to several future load conditions for various design alternatives. If the systemdesign alternative under consideration is not capable of supplying the assumed loads then the set of values for some of the control variables such as voltage levels or power productions; capacitors are adjusted. Much of this time-consuming trial-and error process is reduced by the optimum power flow, and provide a set of feasible values of the control variables. Optimum power-flow solutions may be used not only for system planning but also for system operation, which is a real-time function. In operation, it provides the most economical operating point that meets all the flow and voltage constraints related to power-system security and quality of service. The standard 26 bus test system which has 6 generators, 7 transformers and 9 shunt capacitor is considered in this work.

\section{Problem formulation}

The steady state Optimal Power Flow problem is a minimization problem which is stated as follows. The objective is to minimize the generation cost.

Ravi.C.N. et al /International Journal of ChemTech Research, 2018,11(04): 153-157.

DOI : http://dx.doi.org/10.20902/IJCTR.2018.110418 
Min $\quad C_{t}=\sum_{i=1}^{n g} \alpha_{i}+\beta_{i} P_{i}+\gamma_{i} P_{i}^{2}$

Where, $C_{t}$ - Total cost function, $\alpha, \beta, \gamma$ are cost coefficients, $P_{i}$ is the $i^{\text {th }}$ bus real power generation

Subject To

$$
\begin{array}{lll}
\sum_{i=1}^{n g} P_{i}=P_{D}+P_{L} & \text { (2) } \quad \sum_{i=1}^{n g} Q_{i}=Q_{D}+Q_{L} \\
V_{i(\min )} \leq V_{i} \leq V_{i(\max )} \quad \text { for } \mathrm{i}=1 \text { to Nbus } & \text { (4) } \quad P_{i(\min )} \leq P_{i} \leq P_{i(\max )} & \text { for } \mathrm{i}=1 \text { to } \mathrm{ng} \\
Q_{i(\min )} \leq Q_{i} \leq Q_{i(\max )} \text { for } \mathrm{i}=1 \text { to ng } & \text { (6) } t_{i(\min )} \leq t_{i} \leq t_{i(\max )} \quad \text { for } \mathrm{i}=1 \text { to Ntrans }
\end{array}
$$

Where, $P_{i}, Q_{i}-$ Real and Reactive Power generation of $i^{\text {th }}$ bus, $P_{D}, Q_{D}-$ Real and Reactive Power demand in $i^{\text {th }}$ bus

$\mathrm{P}_{\mathrm{L}}, \mathrm{Q}_{\mathrm{L}}-$ Real and Reactive Power loss, $\mathrm{V}_{\mathrm{i}}-$ Voltage magnitude of the $\mathrm{i}^{\text {th }}$ bus, $\mathrm{t}_{\mathrm{i}}-$ Transformer tap position of the $\mathrm{i}^{\text {th }}$ transformer, Nbus - Number of bus, ng - number of generator Ntrans - number of transformer

Lagrange Function $L=C_{t}+\lambda\left(P_{D}+P_{L}-\sum_{i=1}^{n g} P_{i}\right)+\sum_{i=1}^{n g} \mu_{i(\max )}\left(P_{i}-P_{i(\max )}\right)+\sum_{i=1}^{n g} \mu_{i(\min )}\left(P_{i}-P_{i(\min )}\right)$

Where, $\lambda$ - Lagrange multiplier, $\mu_{\mathrm{i}}-$ Khun-tucker function multiplier

The minimum of this unconstrained function is found at the point where partials of the function to its variables are zero
$\frac{\partial L}{\partial P_{i}}=0$
$\frac{\partial L}{\partial \lambda}=0$
$\frac{\partial L}{\partial \mu_{i(\max )}}=0$
$\frac{\partial L}{\partial \mu_{i(\min )}}=0$

$\mu_{\mathrm{i}(\max )}$ and $\mu_{\mathrm{i}(\min )}$ are zero when Pi is within its limit

From Equation - (9),

$\frac{\partial C_{t}}{\partial P_{i}}+\lambda\left(\frac{\partial C_{t}}{\partial P_{i}}-1\right)=0$ We know that $\frac{\partial C_{t}}{\partial P_{i}}=\frac{\partial C_{i}}{\partial P_{i}}$ since $\mathrm{Ct}=\mathrm{C} 1+\quad \mathrm{C} 2+\ldots . . \mathrm{Cng}$

Therefore $\frac{\partial C_{i}}{\partial P_{i}}+\lambda \frac{\partial P_{L}}{\partial P_{i}}=\lambda \quad$ for $\mathrm{i}=1$ to $\mathrm{ng}$

$\frac{\partial C_{i}}{\partial P_{i}}=\lambda-\lambda \frac{\partial P_{L}}{\partial P_{i}} \frac{\partial C_{i}}{\partial P_{i}}=\lambda\left(1-\frac{\partial P_{L}}{\partial P_{i}}\right) \quad \frac{1}{\left(1-\frac{\partial P_{L}}{\partial P_{i}}\right)} \cdot\left(\frac{\partial C_{i}}{\partial P_{i}}\right)=\lambda \quad \lambda=\frac{\partial C_{i}}{\partial P_{i}} \cdot L_{i}$

Where, Penalty Factor, $L_{i}=\frac{1}{\left(1-\frac{\partial P_{L}}{\partial P_{i}}\right)}$ 


$$
\frac{\partial L}{\partial P_{i}}=2 \sum_{j=1}^{n g} B_{i j} P_{j}+B_{0 i}
$$

From equation 13,

$$
\begin{aligned}
& \beta_{i}+2 \gamma_{i} P_{i}+2 \lambda \sum_{j=1}^{n g} B_{i j} P_{j}+B_{0 i} \lambda=\lambda \\
& \left(\frac{\gamma_{i}}{\lambda}+B_{i i}\right) P_{i}+\sum_{\substack{j=1 \\
j \neq i}}^{n g} B_{i j} P_{j}=\frac{1}{2}\left(1-B_{0 i}-\frac{\beta_{i}}{\lambda}\right)
\end{aligned}
$$

This equation is extended to all generating plants results in following linear equation in matrix form

$$
\left[\begin{array}{ccccc}
\frac{\gamma_{1}}{\lambda}+B_{11} & B_{12} & . & . & B_{1 n g} \\
B_{11} & \frac{\gamma_{2}}{\lambda}+B_{22} & B_{23} & . & B_{2 n g} \\
\cdot & & \cdot & & \cdot \\
\cdot & & & \cdot & \cdot \\
B_{n g 1} & B_{n g 2} & \cdot & \cdot & \frac{\gamma_{n g}}{\lambda}+B_{n g n g}
\end{array}\right]\left[\begin{array}{c}
P_{1} \\
P_{2} \\
\cdot \\
\cdot \\
P_{n g}
\end{array}\right]=\frac{1}{2}\left[\begin{array}{c}
1-B_{01}-\frac{\beta_{1}}{\lambda} \\
1-B_{02}-\frac{\beta_{2}}{\lambda} \\
\cdot \\
\cdot \\
1-B_{0 n g}-\frac{\beta_{n g}}{\lambda}
\end{array}\right]
$$

To find the optimal dispatch for an estimated $\lambda$ the simultaneous linear equation 17 is solved to find $\mathrm{P}$ matrix. Then the iteration process is continued using the gradient method. To do this, from equation $16, \mathrm{Pi}$ at the kth iteration is

$$
P_{i}^{k}=\frac{\lambda^{k}\left(1-B_{0 i}\right)-\beta_{i}-2 \lambda^{k} \sum_{j \neq i} B_{i j} P_{j}^{k}}{2\left(\gamma_{i}+\lambda^{k} B_{i i}\right)}
$$

Substitute equation 19 in equation 10 ,

$$
\sum_{i=1}^{n g} \frac{\lambda^{k}\left(1-B_{0 i}\right)-\beta_{i}-2 \lambda^{k} \sum_{j \neq i} B_{i j} P_{j}^{k}}{2\left(\gamma_{i}+\lambda^{k} B_{i i}\right)}=P_{D}+P_{L}
$$

This can be written as $f(\lambda)=P_{D}+P_{L k}$

Expand the equation 21 L.H.S. using Taylor's series about an operating point $\lambda \mathrm{k}$ and neglecting the higher order terms

$$
\begin{aligned}
& f\left(\lambda^{k}\right)+\frac{\partial f\left(\lambda^{k}\right)}{\partial \lambda} \Delta \lambda^{k}=P_{D}+P_{L}^{k} \\
& \Delta \lambda^{k}=\frac{\Delta P^{k}}{\frac{\partial f\left(\lambda^{k}\right)}{\partial \lambda}} \quad \Delta \lambda^{k}=\frac{\Delta P^{k}}{\sum\left(\frac{\partial P_{i}}{\partial \lambda}\right)^{k}}
\end{aligned}
$$

Where $\quad \sum_{i=1}^{n g}\left(\frac{\partial P_{i}}{\partial \lambda}\right)^{k}=\sum_{i=1}^{n g} \frac{\gamma_{i}\left(1-B_{0 i}\right)-B_{i i} \beta_{i}-2 \gamma_{i} \sum_{j \neq i} B_{i j} P_{j}^{k}}{2\left(\gamma_{i}+\lambda^{k} B_{i i}\right)^{2}}$ 


$$
\lambda^{k+1}=\lambda^{k}+\Delta \lambda^{k} \quad \text { (24) } \Delta P^{k}=P_{D}+P_{L}^{k}-\sum_{i=1}^{n g} P_{i}^{k}
$$

The process is continues until $\Delta \mathrm{P}_{\mathrm{k}}$ is less than a specified accuracy

\section{Test System}

26 bus power system network is considered. Bus 1 is taken as slack bus its voltage adjusted to 1.025 angle $0^{\circ}$ and $\mathrm{P}_{1 \mathrm{Max}}=500 \mathrm{Mw}, \mathrm{P}_{1 \mathrm{Min}}=100 \mathrm{Mw}$, the data for the other generator buses are

\begin{tabular}{|l|c|c|c|c|r|}
\hline $\begin{array}{l}\text { Bus } \\
\text { No }\end{array}$ & $\begin{array}{c}\text { Voltage } \\
\text { Mag. } \\
\text { (pu) }\end{array}$ & $\begin{array}{c}\mathbf{Q}_{\text {Min }} \\
\text { (Mvar) }\end{array}$ & $\begin{array}{c}\mathbf{Q}_{\text {Max }} \\
\text { (Mvar) }\end{array}$ & $\begin{array}{l}\mathbf{P}_{\text {Min }} \\
\text { (Mw) }\end{array}$ & $\begin{array}{l}\mathbf{P}_{\text {Max }} \\
(\mathbf{M w})\end{array}$ \\
\hline 2 & 1.02 & 40 & 250 & 50 & 200 \\
\hline 3 & 1.02 & 40 & 150 & 80 & 300 \\
\hline 4 & 1.05 & 40 & 80 & 50 & 150 \\
\hline 5 & 1.04 & 40 & 160 & 50 & 200 \\
\hline 26 & 1.01 & 15 & 50 & 50 & 120 \\
\hline
\end{tabular}

Transformer Data

\begin{tabular}{|c|c|}
\hline Between Buses & Tap setting (pu) \\
\hline $2-3$ & 0.96 \\
\hline $2-13$ & 0.96 \\
\hline $3-13$ & 1.017 \\
\hline $4-8$ & 1.05 \\
\hline $4-12$ & 1.05 \\
\hline $6-19$ & 0.95 \\
\hline $7-19$ & 0.95 \\
\hline
\end{tabular}

Shunt Capacitive Data

\begin{tabular}{|c|c|}
\hline Buses No & Mvar \\
\hline 1 & 4.0 \\
\hline 4 & 2.0 \\
\hline 5 & 5.0 \\
\hline 6 & 2.0 \\
\hline 9 & 3.0 \\
\hline 11 & 1.5 \\
\hline 12 & 2.0 \\
\hline 15 & 0.5 \\
\hline 19 & 5.0 \\
\hline
\end{tabular}

Generators operating costs in $\$ / \mathrm{h}$

$$
\begin{aligned}
& \mathrm{C}_{1}=240+7.0 \mathrm{P}_{1}+0.007 \mathrm{P}_{1}{ }^{2} \\
& \mathrm{C}_{2}=200+10 \mathrm{P}_{2}+0.0095 \mathrm{P}_{2}{ }^{2} \\
& \mathrm{C}_{3}=220+8.5 \mathrm{P}_{3}+0.009 \mathrm{P}_{3}{ }^{2} \\
& \mathrm{C}_{4}=200+11 \mathrm{P}_{4}+0.009 \mathrm{P}_{4}{ }^{2} \\
& \mathrm{C}_{5}=220+10.5 \mathrm{P}_{5}+0.008 \mathrm{P}_{5}{ }^{2} \\
& \mathrm{C}_{26}=190+12 \mathrm{P}_{26}+0.0075 \mathrm{P}_{26}{ }^{2}
\end{aligned}
$$




\section{Test Result}

Test result for the 26 bus power system is summarized in the following table

\begin{tabular}{|l|c|c|c|}
\hline \multicolumn{1}{|c|}{ Description } & $\begin{array}{c}\text { Base Case } \\
\text { Power } \\
\text { Flow }\end{array}$ & $\begin{array}{c}\text { Optimal Power } \\
\text { Flow }\end{array}$ & Savings \\
\hline Total Generation Cost $(\$ / \mathrm{h})$ & 16760.73 & 15447.72 & 1313.01 \\
\hline Total system loss $(\mathrm{Mw})$ & 15.53 & 12.807 & 2.723 \\
\hline & 474.1196 & 447.6919 & \\
System Power Generation & 173.7886 & 173.1938 & \\
by the generators $(\mathrm{Mw})$ & 190.9515 & 263.4859 & \\
& 150.0000 & 138.8142 & \\
& 196.7196 & 165.5884 & \\
\hline Incremental cost of & 103.5772 & 87.0260 & \\
delivered power $(\lambda)$ & 13.911780 & 13.538113 & \\
(\$/MWh) & & & \\
\hline
\end{tabular}

\section{Conclusion}

The optimum power flow has been defined and its advantages over the ordinary power flow have been shown to be greatly reduced trial and error. It has been shown that Newton's method of power flow solution can be extended to yield an optimal power flow solution that is feasible with respect to all relevant inequality constraints. The main features of the method are a gradient procedure for finding the optimum and the use of penalty functions to handle functional inequality constraints.

\section{References}

1. H. W. Dommel \& W. F. Tinney, "Optimal power flow solutions", IEEE Trans. (Power App. \& Syst.), Vol. PAS-87, October, 1968 ,pp. 1866-1876.

2. Allen J. Wood and Bruce F. Wollenberg, Power Generation, Operation, and Control, John Wiley \& Sons, Inc.

3. Kundur P, 'Power System Stability and Control', Mc Graw Hill Publication,

4. O. Alsac and B. Stott, "Optimal Load Flow with Steaty State Security", IEEE power system engineering conference

5. Ravi. C N, Christober Asir Rajan C Ravi, C. N. "Optimal power flow solutions using constraint Genetic Algorithm." National Journal on Advances in Computing and Management 3.1 (2012).

6. Ghaddar, Bissan, Jakub Marecek, and Martin Mevissen. "Optimal power flow as a polynomial optimization problem." IEEE Transactions on Power Systems 31.1 (2016): 539-546.

7. Bazrafshan, Mohammadhafez, and Nikolaos Gatsis. "Decentralized stochastic optimal power flow in radial networks with distributed generation." IEEE Transactions on Smart Grid 8.2 (2017): 787-801. 